\title{
Numerical Analysis of Thermal and Hydro Dynamical Processes in Upper Fuel Channel Part of Boiling Water Reactor
}

\author{
Linas PAUKŠTAITIS*, Sigitas KILIKEVIČIUS**, Valdas LUKOŠEVIČIUS***, Algimantas \\ FEDARAVIČIUS****, Marius GUDAUSKIS*****, Algimantas BALČIUS******, Juozas \\ GUDZINSKAS******** \\ *Kaunas University of Technology, Studentu 56, LT-51424, Kaunas, Lithuania, E-mail: linas.paukstaitis@ktu.lt \\ **Kaunas University of Technology, Studentu 56, LT-51424, Kaunas, Lithuania, E-mail: sigitas.kilikevicius@ktu.lt \\ ***Kaunas University of Technology, Studentu 56, LT-51424, Kaunas, Lithuania, E-mail: valdas.lukosevicius@ktu.lt \\ ****Kaunas University of Technology, Studenty 56, LT-51424, Kaunas, Lithuania, \\ E-mail: algimantas.fedaravicius@ktu.lt \\ *****Kaunas University of Technology, Studentu 56, LT-51424, Kaunas, Lithuania, E-mail: marius.gudauskis@ktu.lt \\ ******Kaunas University of Technology, Studentu 56, LT-51424, Kaunas, Lithuania, E-mail: algimantas.balcius@ktu.lt \\ *******Kaunas University of Technology, Studentu 56, LT-51424, Kaunas, Lithuania, E-mail: juozas.gudzinskas@ktu.lt \\ crossref http://dx.doi.org/10.5755/j01.mech.24.1.19468
}

\section{Introduction}

The RBMK-1500 reactor [1] was the last modification of the modernized soviet channel type reactor RBMK-1000 [2]. Fig. 1 shows a general view of the fuel assembly of the RBMK-1500. The upper part (upper bundle) of the fuel assembly is shown on the right side in Fig. 1. More details concerning the design features of the RBMK reactor are presented in the paper [3].

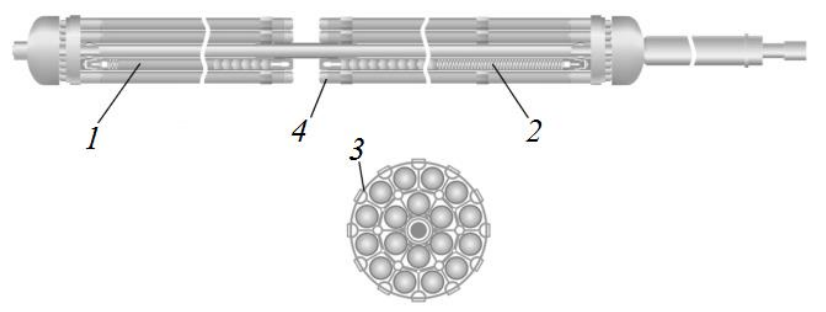

Fig. 1 Fuel assembly of the reactor RBMK-1500 (drawn by Panther) [1, 2] 1 - lower bundle; 2 - upper bundle; 3 - spacer; 4 - fuel rod

The main problem, which occurred for the designers of the RBMK-1500 reactor core, was how to increase (by 1.5 times) the power of the RBMK-1000 reactor without increasing the reactor's core size. Such a task was only possible to reach by increasing the power of the separate fuel channel. However, the augmentation of the power influenced on a rapid boost of steam generation, and as a sequence, on the formation of dry out (film boiling or the second boiling crisis) [4-7]. That problem was solved by using special heat transfer intensification grids-intensifiers (Fig. 2) in the upper part of the fuel assembly, where steam generation was the most intensive. In total the upper part of the fuel assembly (upper bundle) has 10 distance grids-spacers (Fig. 2, a) and 30 heat transfer intensification grids-intensifiers (Fig. 2, b).

Heat transfer intensification grids together with the distance grids increased a turbulence of the water-steam flow and intensified the heat transfer rate between the fuel elements and the coolant (water-steam mixture) [8,9]. However, the additional obstacles (spacers and intensifiers) created much more complicated distribution of the velocity, temperature, void fraction and etc. An experimental investigation of such parameters on the operating reactor was very complicated. Therefore, a numerical analysis of the thermal and hydro dynamical processes, which occurred during the coolant flow inside the upper part of the fuel channel, was very important for the safe operation of the reactor.

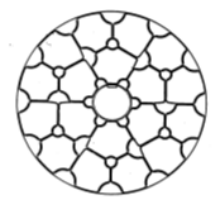

a

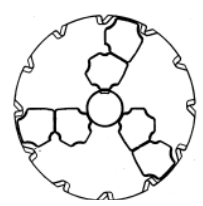

b
Fig. 2 Spacer (a) and intensifier (b) of the fuel assembly of the reactor RBMK-1500 [10]

A numerical investigation of the thermal and hydro dynamical processes which take place inside the fuel channel of the RBMK-1500 reactor during interaction between the coolant and the fuel assembly, was presented in [3]. Thermal and hydro dynamical processes in the numerical model were estimated at the 13 points of the technological channel's cross section (Fig. 3).

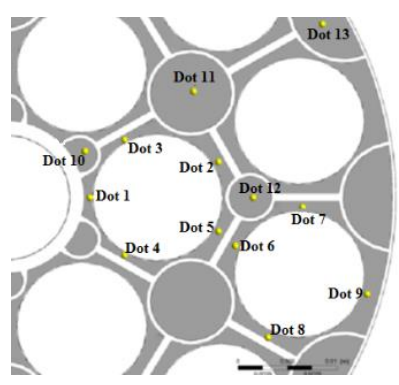

Fig. 3 Position of the check points in the cross section of the channel [3]

The investigation covered the estimation of the coolant's temperature distribution along and across the channel, localization of the steam generation places, determination of the coolant velocity, steam fraction distribution and etc. 
This paper presents a numerical investigation of the thermal and hydro dynamical processes, which take place inside the upper part of the fuel channel during the hydro and thermal interaction between the coolant and the fuel bundles.

\section{Variation of parameters along the fuel channel}

The numerical investigation was carried out according the methodology used in the preceding investigation presented in paper [3], under the same conditions. Accordingly, the coolant parameters at the inlet to the fuel channel were set as follow: the temperature of $263.8^{\circ} \mathrm{C}$; the pressure of $7 \mathrm{MPa}$; the flow rate of $5.12 \mathrm{~kg} / \mathrm{s}$ and the power of the fuel assembly was $2.605 \mathrm{MW}$.

Fig. 4 presents the change of the coolant temperature in the upper part of the fuel assembly at the height of 5.95-6.65 $\mathrm{m}$ of the fuel channel at the points, which correspond to the ones showed in Fig. 3.

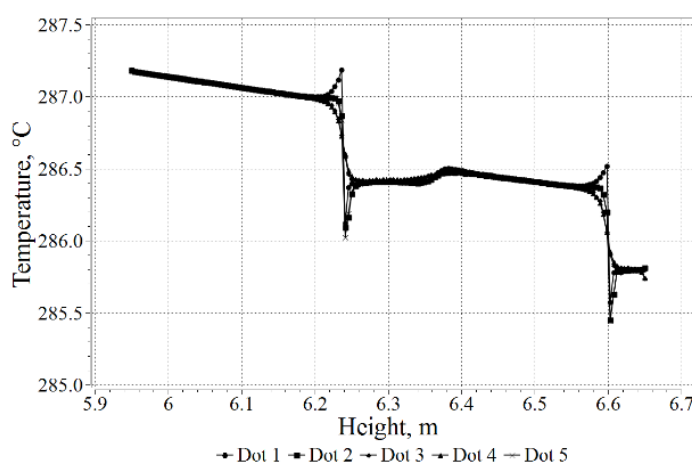

a

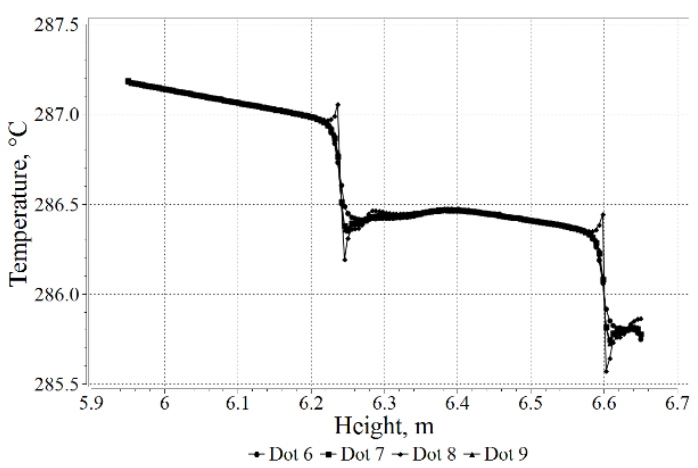

$\mathrm{b}$

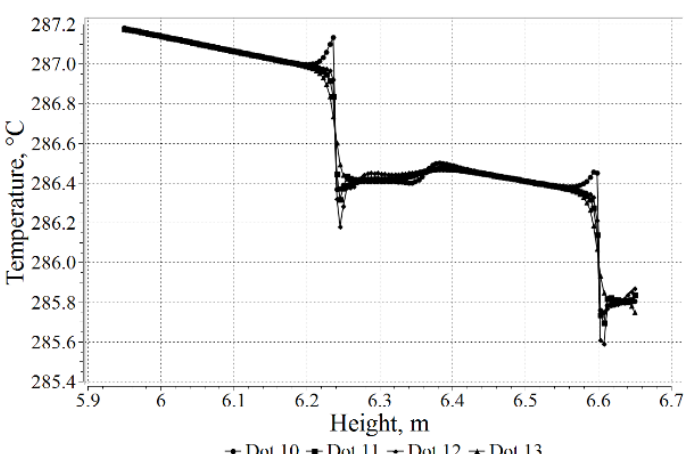

c

Fig. 4 Temperature change along the fuel channel: (a) at the $1-5$ points; (b) at the $6-9$ points; (c) at the $10-13$ points
The heat, which was released in the lower part of the channel, was utilized for the water heating and evaporation, while the heat, generated in the upper part of the channel, was used for water evaporation only. Consequently, an intensive steam generation and fully developed two phase (water-steam) flow were observed. The saturation temperature of the coolant was reducing due to the reduction of the coolant's pressure (Fig. 7) and the increase of the velocity (Fig. 6). The temperature decrease was particularly steep at the intensification grids because of the pressure drop.

Fig. 5 demonstrates the change of the steam mass fraction in upper part of the fuel assembly.
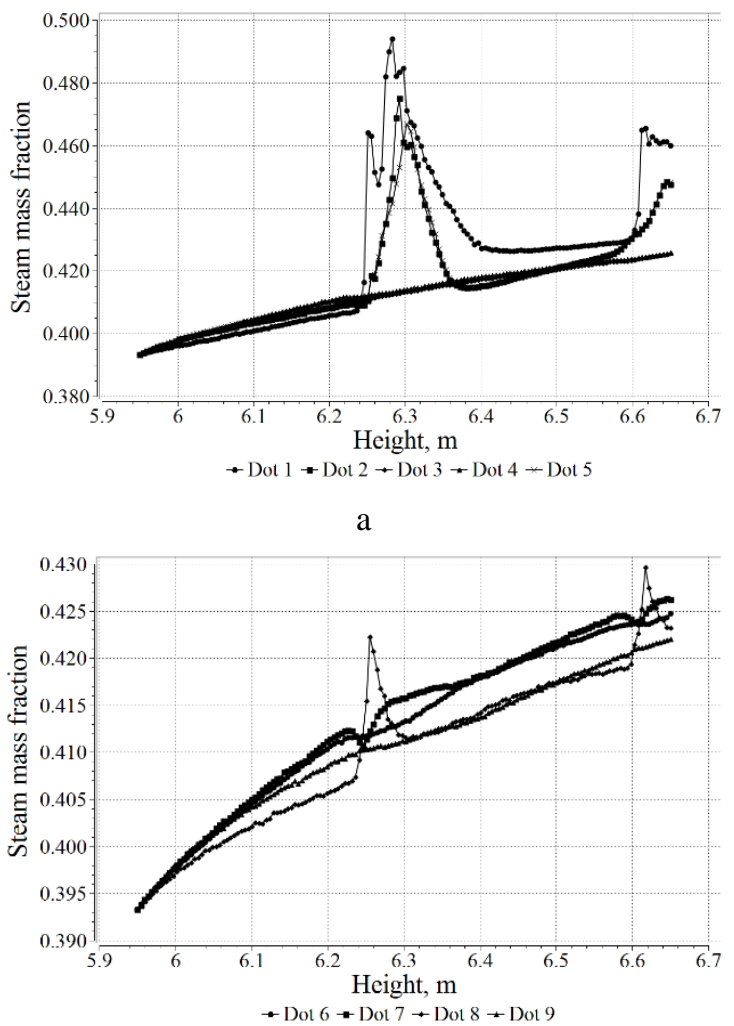

b



Fig. 5 Steam mass fraction change along the fuel channel: (a) at the 1-5 points; (b) at the 6-9 points; (c) at the 10-13 points

The steam generation in that part of the fuel assembly was more intensive due to the influence of the heat transfer intensification grids. The increase of the turbulence of the coolant (water-steam mixture) acted on the grow of the heat transfer rate and on the intensity of the steam generation. Steam fraction increased at the points 1, 2, 5, 8 and 10 
due to the reduction of the fuel channel cross-section and the reduction of the pressure.

Fig. 6 presents the change of the coolant velocity in upper part of the fuel assembly.

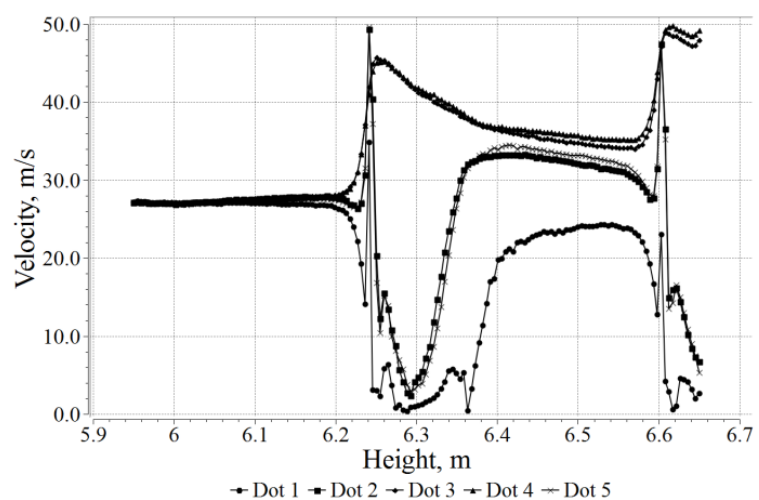

a

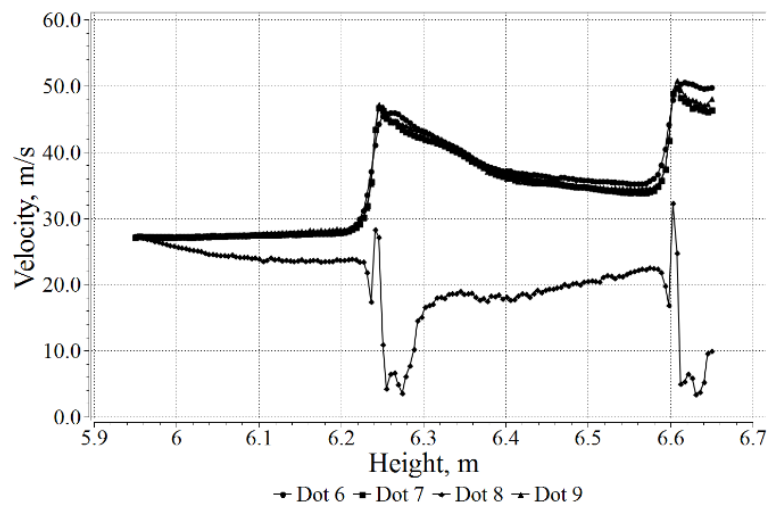

b

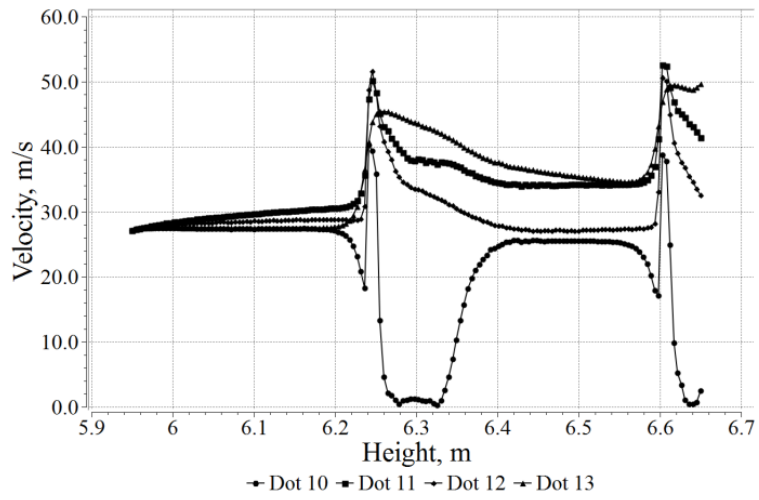

c

Fig. 6 Flow velocity change along the fuel channel; (a) at the 1-5 points; (b) at the 6-9 points; (c) at the 10-13 points

The regularities of the velocity change in the upper part of the fuel channel were similar like in the lower part of the channel. Although, the differences were observed: an average flow velocity was much higher in the upper part (by about $30 \mathrm{~m} / \mathrm{s}$ ) due to the flow of the two phase coolant.

Fig. 7 presents the change of the coolant pressure in the upper part of the fuel assembly.

The pressure loss in the upper part of the channel was higher in comparison with the lower part of the channel due to the high amount and close proximity of the heat transfer intensification grids intensifiers. The higher coolant velocity also influenced the more rapid pressure reduction.

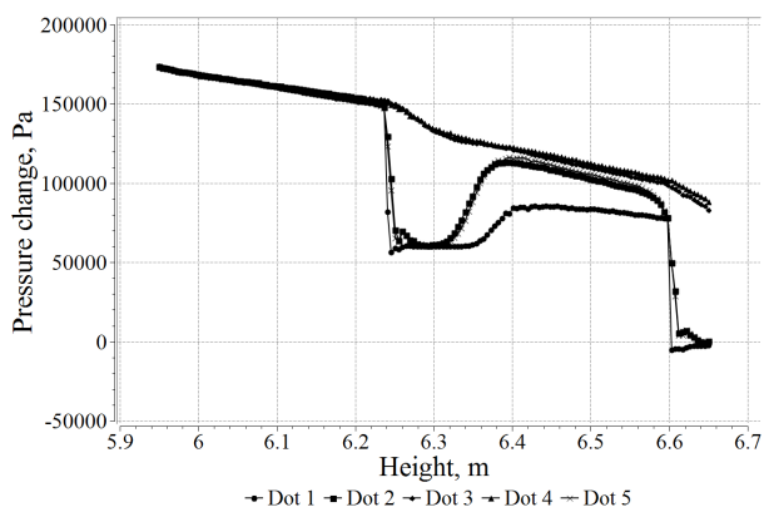

a

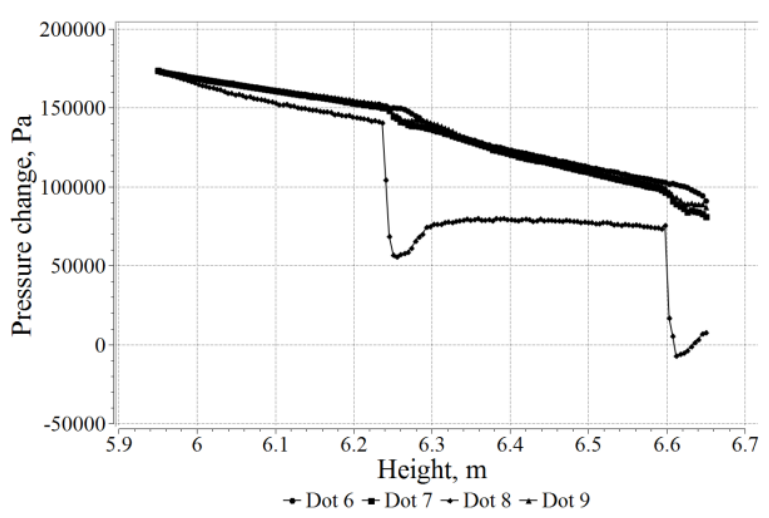

$\mathrm{b}$

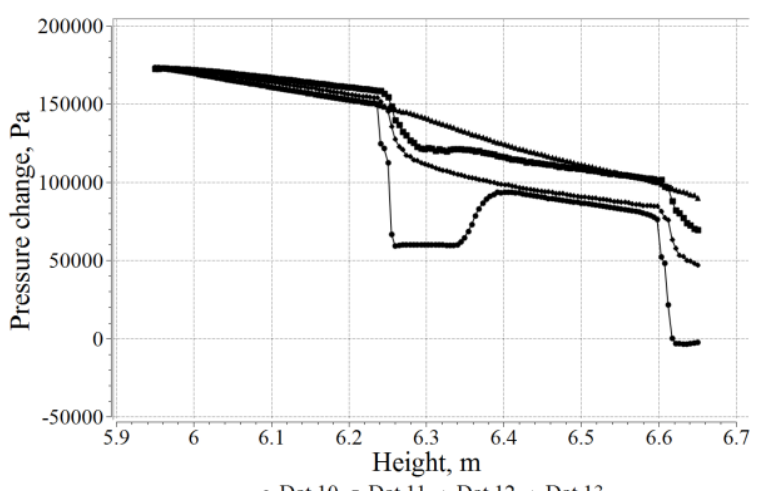

C

Fig. 7 Flow pressure change along the fuel channel: (a) at the $1-5$ points; (b) at the 6-9 points; (c) at the 10-13 points

Fig. 8 shows the distribution of the heat transfer coefficient along the upper part of the fuel channel. The average value of the heat transfer coefficient was equal to $40000 \mathrm{~W} /\left(\mathrm{m}^{2} \cdot \mathrm{K}\right)$ approximately (about $14 \%$ higher). The local increase of the heat transfer coefficient was influenced by the heat transfer intensification grids due to the reduction of the free cross-sectional area of the channel as well as the increase of the turbulence of the coolant flow.

As it was shown in the preceding paper [3], the heat transfer coefficient in the lower part of the fuel channel increased at the spacer and returned back after it. Meanwhile, in the upper part of the fuel channel, the heat transfer coefficient increased at the intensifier but did not return back after it. The main reason of that was the higher turbulence of the water-steam mixture and intensive vortex formation. All those processes influenced on the more intensive wetting of 
the fuel elements by the water droplets and, as a sequence, on more intensive evaporation of the droplets.

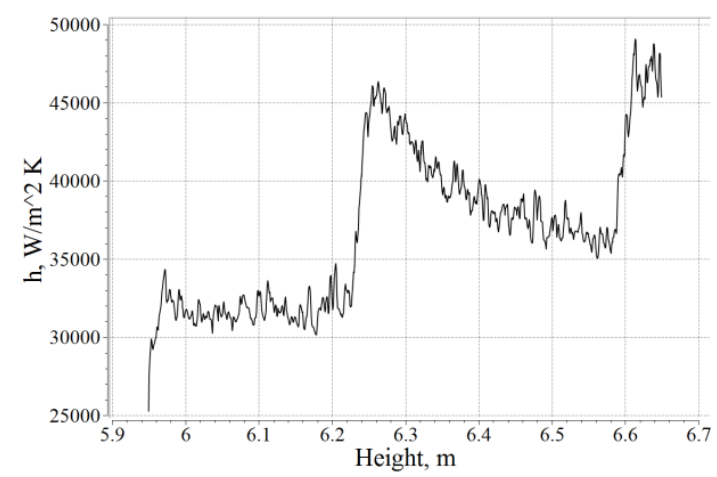

Fig. 8 Distribution of the heat transfer coefficient along the upper part of the fuel channel at the $5.95-6.65 \mathrm{~m}$ height

\section{Variation of parameters across the fuel channel}

Figs. 9-13 represent the variation of the thermal and hydro dynamical parameters at the cross-sections of the upper part of the fuel channel at the height equal to $6.595 \mathrm{~m}$ (before the intensifier) and at the height of $6.605 \mathrm{~m}$ (at the intensifier).
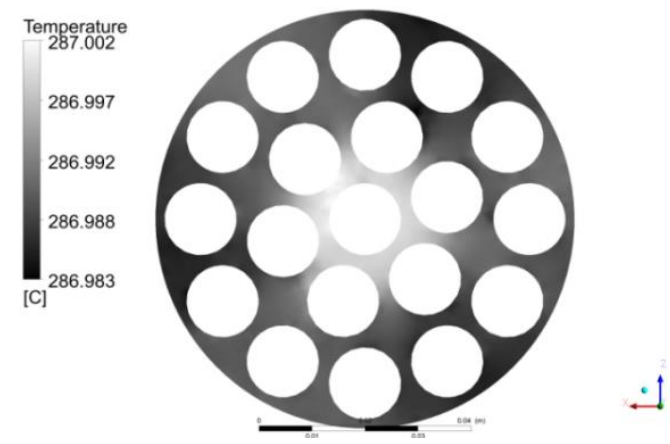

a
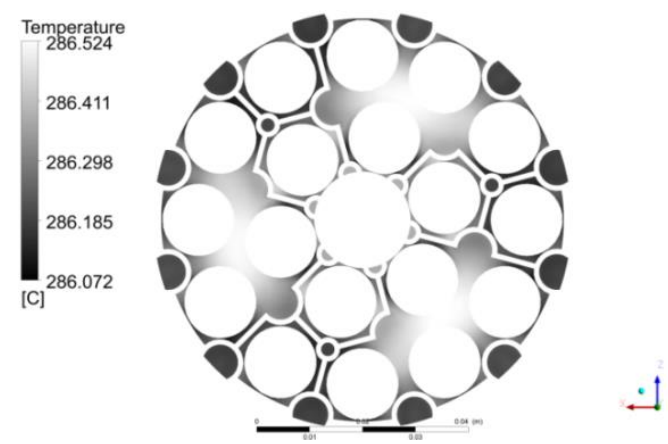

$\mathrm{b}$

Fig. 9 Coolant temperature distribution across the fuel bundle: (a) fuel channel without the intensifier; (b) with the intensifier

Fig. 9 presents the coolant temperature distribution in the upper part of the fuel assembly. Coolant temperature was almost uniform in the channel without the intensifier. The temperature distribution in the channel with the intensifier was influenced by the grid's special shape which increased the turbulence of the flow.
Fig. 10 shows the steam mass fraction distribution in the upper part of the fuel assembly. The steam mass fraction distribution in the channel without the intensifier was similar to the distribution in the lower part of the fuel channel. The slight difference could be explained as the result of the influence of the intensifier, which was placed below the treated cross-section shown in the picture. The steam mass fraction distribution at the intensifier was determined by the turbulence of the flow which carried the steam towards the central part of the channel.
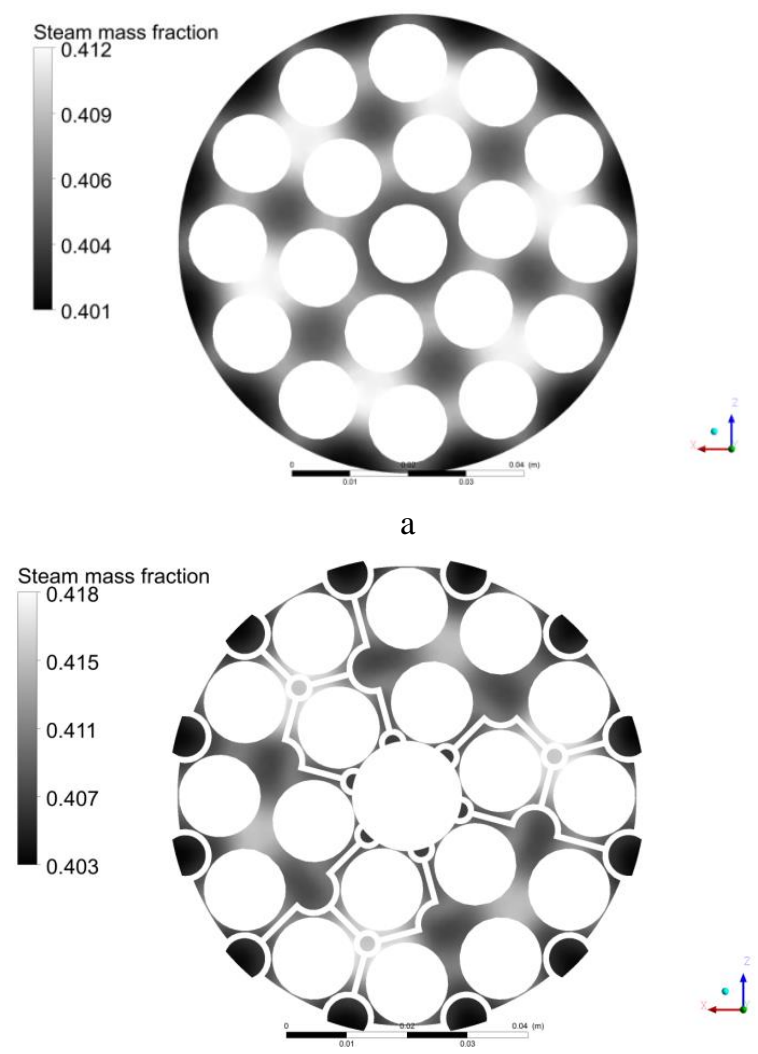

b

Fig. 10 Steam mass fraction distribution across the fuel bundle: (a) fuel channel without the intensifier; (b) with the intensifier

Fig. 11 presents the coolant velocity distribution before the intensifier and at the intensifier in the upper part of the fuel assembly. Character of the velocity distribution was similar to the distribution in the lower part of the fuel channel. However, the velocity was much higher in the upper part of the channel due to the presence of the steam in the coolant flow. It was possible to observe six peaks of the velocity in the lower part of the channel. However, the velocity of coolant was more uniform in the upper part of the channel where only three maximums of the velocity were observed. The special shape of the intensifier influenced the rotation of the coolant clockwise.

Fig. 12 shows the coolant pressure distribution in the cross section area of the fuel channel before the intensifier and with the intensifier in the upper part of the fuel assembly. The pressure distribution was almost uniform before the grid in the cross section of the channel because of the coolant's temperature, which reached the saturation temperature and the heat was used for the evaporation only. The pressure was reduced at the intensifier due to the grid's resistance. 

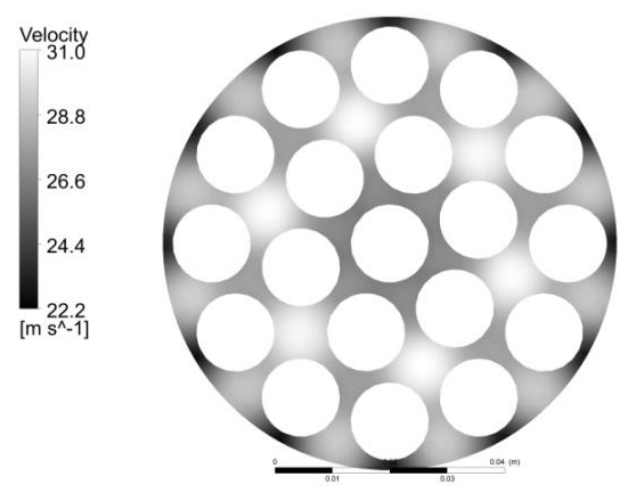

a
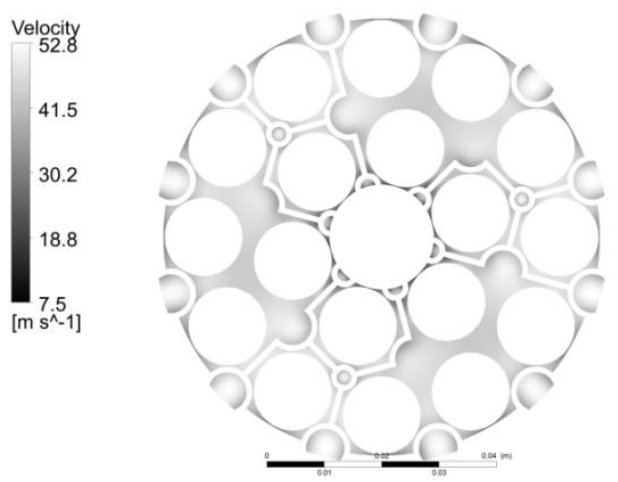

b

Fig. 11 Coolant velocity distribution across the fuel bundle: (a) fuel channel without the intensifier; (b) with the intensifier



a

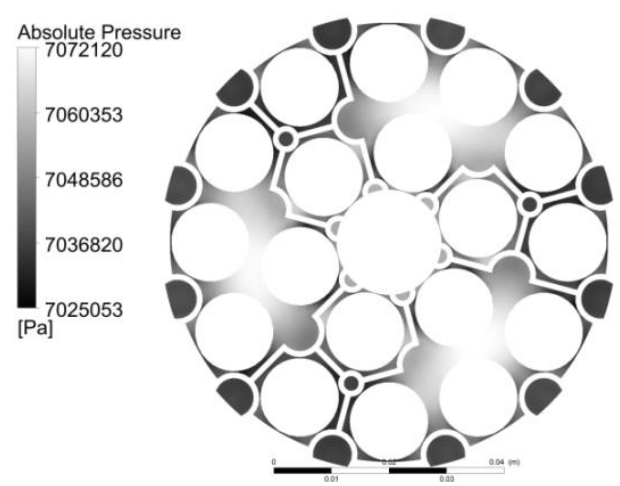

b

Fig. 12 Coolant pressure distribution across the fuel bundle: (a) fuel channel without the intensifier; (b) with the intensifier
Fig. 13 presents the steam void fraction distribution in the different cross sections of the upper part of the fuel assembly. The significant influence of the intensifiers on the steam generation process was observed. That influence of the grids was especially visible for the steam mass fraction alteration between the second and the third intensifiers. The steam mass fraction was higher in the central part of the channel due to the turbulence induced by the special shape of the intensifiers.

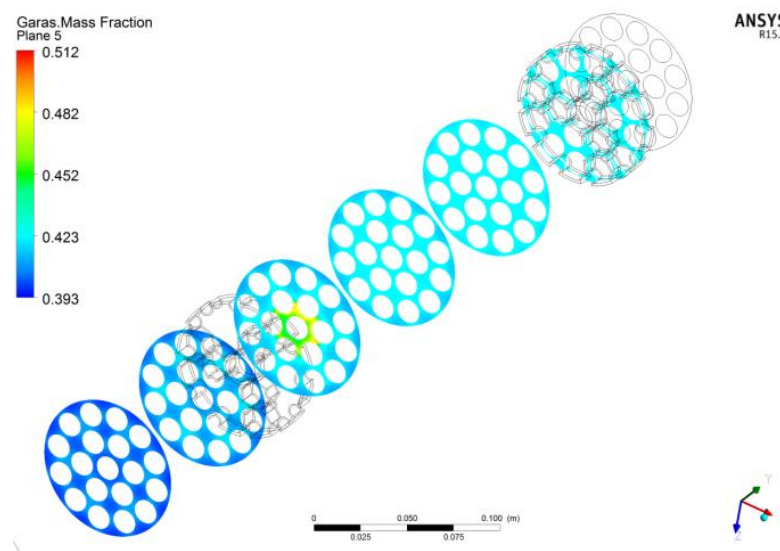

Fig. 13 Steam mass fraction distribution in the different cross sections of the upper part of the fuel bundle

\section{Conclusions}

The coolant temperature was almost uniform in the channel without the intensifier. Temperature distribution in the channel with the intensifier was influenced by the grid's special shape increasing a turbulence of the flow. The temperature decrease was particularly steep at the grid due to the sudden pressure drop. The saturation temperature of the coolant was reduced due to the reduction of the coolant's pressure as well as the increase of the two-phase flow velocity.

The character of the velocity distribution in the upper part of the fuel channel was similar to the distribution in the lower part of the fuel channel. However, the average velocity was much higher (by about $30 \mathrm{~m} / \mathrm{s}$ ) and more uniform in the upper part of the channel due to the presence of the steam in the coolant flow. The special shape of the intensifiers forced the coolant to rotate clockwise.

The pressure loss in the upper part of the channel was higher in comparison with the lower part of the channel due to the high amount and close proximity of the intensifiers. The higher coolant velocity also influenced the more rapid pressure reduction. The pressure distribution was almost uniform before the grid in the cross section of the channel because of the coolant temperature, which reached the saturation temperature and the heat was used for the evaporation only. The pressure was reduced at the grid due to the grid's resistance.

The steam mass fraction was higher in the central part of the channel due to the turbulence induced by the special shape of the intensifiers. The distribution in the channel without the intensifiers was similar to the distribution in the lower part of the channel. The slight difference could be explained by the influence of the previous intensifier placed below the treated cross section. 
The heat, which was generated in the upper part of the channel, was used for water boiling (evaporation) only; consequently, fully developed two phase (water-steam) flow was observed. Increase of the turbulence of the coolant (water-steam mixture) affected on the grow of the heat transfer rate and on the intensity of the steam generation. The average value of the heat transfer coefficient in the upper part of the channel was equal to $40000 \mathrm{~W} /\left(\mathrm{m}^{2} \cdot \mathrm{K}\right)$ approximately (about $14 \%$ higher than in the lower part of the channel).

The local growth of the heat transfer coefficient was influenced by the intensifiers due to the reduction of the free cross-sectional area of the channel and because of the increase of the turbulence of the coolant flow. The heat transfer coefficient in the lower part of the fuel channel increased at the spacers and returned back after the grids. Meanwhile, in the upper part of the fuel channel, the heat transfer coefficient increased at the intensifiers but did not return back after them. The main reason of that was the turbulence of the water-steam mixture and vortex formation. All those processes have influenced on the more intensive wetting of the fuel elements by the water droplets and on the more intensive evaporation of the droplets.

\section{References}

1. Almenas, K.; Kaliatka, A.; Ušpuras, E. 1998. Ignalina RBMK-1500. A Source Book. Kaunas: Lithuanian Energy Institute, 198 p.

2. Abramov, M.I.; Avdeev, V.I.; Adamov, E.O. et al. 2006. Channel type nuclear energy reactor RBMK1000. GUP NIKIET, 632 p. (in Russian).

3. Paukštaitis, L.; Kilikevičius, S.; Gudzinskas, J.; Gylys, M.; Lukoševičius, V. 2017. Numerical analysis of thermal and hydro dynamical processes in lower fuel channel part of boiling water reactor. Mechanika, 23(5): 653-660. http://dx.doi.org/10.5755/j01.mech.23.5.16457.

4. Jouhara, H. 2003. Film boiling heat transfer on spherical, cylindrical and plane geometries. Ph.D. Thesis, University of Manchester.

5. Burns, R.A. 1989. Heat Transfer Studies with Application to Nuclear Reactors. Ph.D. Thesis, University of Manchester.

6. Situ, R.; Hibiki, T.; Sun, X.; Mi, Y.; Ishii, M. 2004. Flow structure of subcooled boiling flow in an internally heated annulus. International Journal of Heat and Mass Transfer, 47(24): 5351-5364.

https://doi.org/10.1016/j.ijheatmasstransfer.2004.06.035.

7. In, W.K.; Hwang, D.H.; Jeong, J.J. 2013. A subchannel and CFD analysis of void distribution for the BWR fuel bundle test benchmark. Nuclear Engineering and Design, 258, 211-225. https://doi.org/10.1016/j.nucengdes.2013.02.006.

8. Jayanti, S.; Reddy, K.R. 2013. Effect of spacer grids on CHF in nuclear rod bundles. Nuclear Engineering and Design, 261: 66-75. https://doi.org/10.1016/j.nucengdes.2013.03.044.

9. Renksizbulut, M.; Hadaller, G.I. 1986. An experimental study of turbulent flow through a square-array rod bundle. Nuclear engineering and design, 91(1): 4155.

https://doi.org/10.1016/0029-5493(86)90183-4.

10. Stravinskas, S.; $\quad$ Pashkevichius, G.; Krivov, I.; Krivoshein, G. Equipment for repair of the RBMK1500 fuel assemblies of Ignalina NPP http://www.dysnai.org/Reports/2000.

L. Paukštaitis, S. Kilikevičius, V. Lukoševičius,

A. Fedaravičius, M. Gudauskis, A. Balčius, J. Gudzinskas

NUMERICAL ANALYSIS OF THERMAL AND HYDRO DYNAMICAL PROCESSES IN UPPER FUEL CHANNEL PART OF BOILING WATER REACTOR

S u m m a r y

This article is the second part of the $3 \mathrm{D}$ numerical analysis of the thermal and hydro dynamical processes which take place inside the fuel channel of the RBMK-1500 reactor during the interaction between the coolant and the fuel bundles. This article covers the results devoted to the upper part of the fuel channel, meanwhile, the preceding investigation [3] focuses on the processes, which run inside the lower part of the fuel channels of the RBMK-1500 reactor.

The evaluation of the pressure drop, coolant temperature rise, flow velocity and steam mass fraction changes at the distance grids (spacers) and at the heat transfer intensification grids-intensifiers of the upper part of the fuel channel was carried out using the ANSYS CFX software.

It was stated that the heat transfer intensification grids (intensifiers) increased the turbulence of the coolant (water-steam mixture) and influenced on the rise of the heat transfer rate and on the intensity of the steam generation. The average value of the heat transfer coefficient in the upper part of the channel was equal to $40000 \mathrm{~W} /\left(\mathrm{m}^{2} \cdot \mathrm{K}\right)$ and was about $14 \%$ higher than in the lower part of the channel. Differently from the lower part of the channel, the heat transfer coefficient increased at the heat transfer intensification grids and did not return back to the previous values after passing through them. The main reason of that was the turbulence of the water-steam mixture and vortex formation. All those processes have influenced on the more intensive wetting of the fuel elements by the water droplets and on the more intensive droplets evaporation.

Keywords: fuel channel; coolant temperature; velocity; pressure drop; steam fraction; spacers; intensifiers.

Received November 11, 2017 Accepted February 15, 2018 